\title{
Cuerpo extraño en el esófago como causa de síntomas respiratorios en el niño. Casos clínicos
}

\author{
Foreign body in the esophagus as a cause of respiratory symptoms in children. \\ Clinical cases
}

\author{
Dra. Giselle Cuestas ${ }^{a}$, Dra. Verónica Rodrígueza , Dra. Flavia Doormann ${ }^{a}$ Dr. Patricio Bellia Munzón $n^{a}$ y \\ Dr. Gastón Bellia Munzón ${ }^{b}$
}

\begin{abstract}
RESUMEN
Los cuerpos extraños en el esófago son accidentes evitables que se observan, con mayor frecuencia, en niños menores de 3 años. Los síntomas de presentación más comunes son la disfagia, la sialorrea y los vómitos. Ocasionalmente, un cuerpo extraño en el esófago puede manifestarse con síntomas respiratorios, tales como tos, estridor y dificultad respiratoria. Esto es más frecuente en los niños pequeños y cuando el objeto permanece alojado en el esófago durante un período prolongado.

La sospecha clínica es fundamental para el diagnóstico precoz, lo cual permite evitar potenciales complicaciones.

Describimos a 3 niños con un cuerpo extraño impactado en el esófago que presentaron, principalmente, síntomas respiratorios. Alertamos a los pediatras sobre la variación sintomática en la presentación de un cuerpo extraño ingerido y subrayamos la importancia de realizar un diagnóstico y tratamiento oportunos.

Palabras clave: cuerpos extraños, esófago, signos y síntomas respiratorios, niño.
\end{abstract}

\begin{abstract}
Foreign bodies in esophagus are avoidable accidents that occur most often in children younger than 3 years.

The most common presenting symptoms are dysphagia, drooling and vomiting. Occasionally a foreign body in the esophagus may present with respiratory symptoms such as cough, stridor and respiratory distress. This is more common in young children and when the object remains lodged in the esophagus for a prolonged period.

Clinical suspicion is essential for early diagnosis, which allows to avoid potential complications.

We describe 3 children with a foreign body impacted in the esophagus who presented mainly respiratory symptoms. We alert pediatricians on symptomatic variation in the presentation
\end{abstract}

a. Sección de Endoscopía Respiratoria, División de Otorrinolaringología, Hospital General de Niños “Dr. Pedro de Elizalde", Ciudad Autónoma de Buenos Aires, Argentina.

b. Servicio de Cirugía, Hospital General de Niños "Dr. Pedro deElizalde", Ciudad Autónoma de Buenos Aires, Argentina.

Correspondencia:

Dra. Giselle Cuestas, giselle_cuestas@yahoo.com.ar

Financiamiento: Ninguno.

Conflicto de intereses: Ninguno que declarar.

Recibido: 3-11-2016

Aceptado: 30-11-2016 of a foreign body ingestion and we underline the importance of early diagnosis and treatment.

Key words: foreign bodies, esophagus, respiratory signs and symptoms, child.

http:/ / dx.doi.org/10.5546/aap.2017.e126

Cómo citar: Cuestas G, Rodríguez V, Doormann F., et al. Cuerpo extraño en el esófago como causa de síntomas respiratorios en el niño. Casos clínicos. Arch Argent Pediatr 2017;115(2):e126-e130.

\section{INTRODUCCIÓN}

La mayoría de los cuerpos extraños (CE) ingeridos atraviesan el tubo digestivo sin ocasionar manifestaciones clínicas ni complicaciones; sin embargo, un porcentaje significativo se impacta en el esófago y causa, típicamente, sialorrea, odinofagia y dificultad en la alimentación (rechazo de la comida, eliminación de sólidos en la dieta). ${ }^{1,2}$

Con menor frecuencia, un CE alojado en el esófago puede presentarse con síntomas respiratorios, principalmente, con tos y estridor, en lugar de síntomas gastrointestinales. Esta situación es más común cuando el CE está presente por un período prolongado (más de 1 semana), es grande y comprime la tráquea, y en los niños menores de 3 años. ${ }^{3}$

La radiografía simple de tórax puede confirmar el diagnóstico, ya que la mayoría de los CE esofágicos son radiopacos. Cuando no hay testigos del episodio de atoramiento y el CE es radiolúcido, el diagnóstico puede ser difícil. ${ }^{4,5}$ En estos casos, la radioscopía con ingesta de sustancia baritada puede ponerlo de manifiesto. ${ }^{1,6}$

La sospecha clínica y la investigación radiológica adecuada son fundamentales para el diagnóstico precoz. El retraso de este último empeora el pronóstico, ya que da lugar a posibles complicaciones, como perforación o estenosis esofágica. ${ }^{1}$ La endoscopía rígida bajo anestesia general es el método de elección para extraer los objetos retenidos en el esófago en los niños. ${ }^{1,6}$ 


\section{CASOS CLÍNICOS}

Entre el año 2013 y el 2016, tres niños con CE esofágico fueron derivados a la Sección de Endoscopía Respiratoria del Hospital General de Niños "Dr. Pedro de Elizalde". El síntoma principal de presentación en los 3 casos fue la tos de varias semanas de evolución (Tabla 1). Uno de ellos presentaba, además, estridor bifásico (inspiratorio y espiratorio) intenso. En otras instituciones, recibieron tratamiento médico por bronquitis, sin respuesta.

Dos de los pacientes no presentaban dificultades en la deglución ni sialorrea; el restante había eliminado los sólidos de la dieta.
No tenían antecedentes perinatales patológicos. Ningún paciente presentó historia de ingestión de CE.

La radiografía simple de tórax, de frente y de perfil, reveló, en los 3 casos, un CE radiopaco en el esófago que ocasionaba una disminución del calibre traqueal (Figuras 1 y 2).

Se realizó laringoscopía directa y esofagoscopía rígida bajo anestesia general, y se evidenció la compresión de la pared posterior de la tráquea y el objeto metálico alojado a nivel del músculo cricofaríngeo (Figura 3). En cada caso, se extrajo el CE con una pinza de agarre.

Los pacientes evolucionaron sin complicaciones

Tabla 1. Características de los pacientes

\begin{tabular}{lcccccc}
\hline Paciente & Sexo & Edad & Duración de los síntomas & Síntomas principales & Disfagia & Cuerpo extraño \\
\hline 1 & Masculino & 15 meses & 3 semanas & Tos y estridor & No & Arandela \\
2 & Femenino & 18 meses & 1 mes & Tos & No & Dije \\
3 & Masculino & 4 años & 1 mes & Tos & Sí & Moneda \\
\hline
\end{tabular}

FIgURA 1. Radiografía simple de tórax. Vista anteroposterior. Cuerpo extraño radiopaco en el tercio superior del esófago (flecha). Vista lateral. Compresión traqueal (flecha). A. Paciente $n^{\circ}$ 1. Arandela. B. Paciente $n^{\circ} 2$. Dije

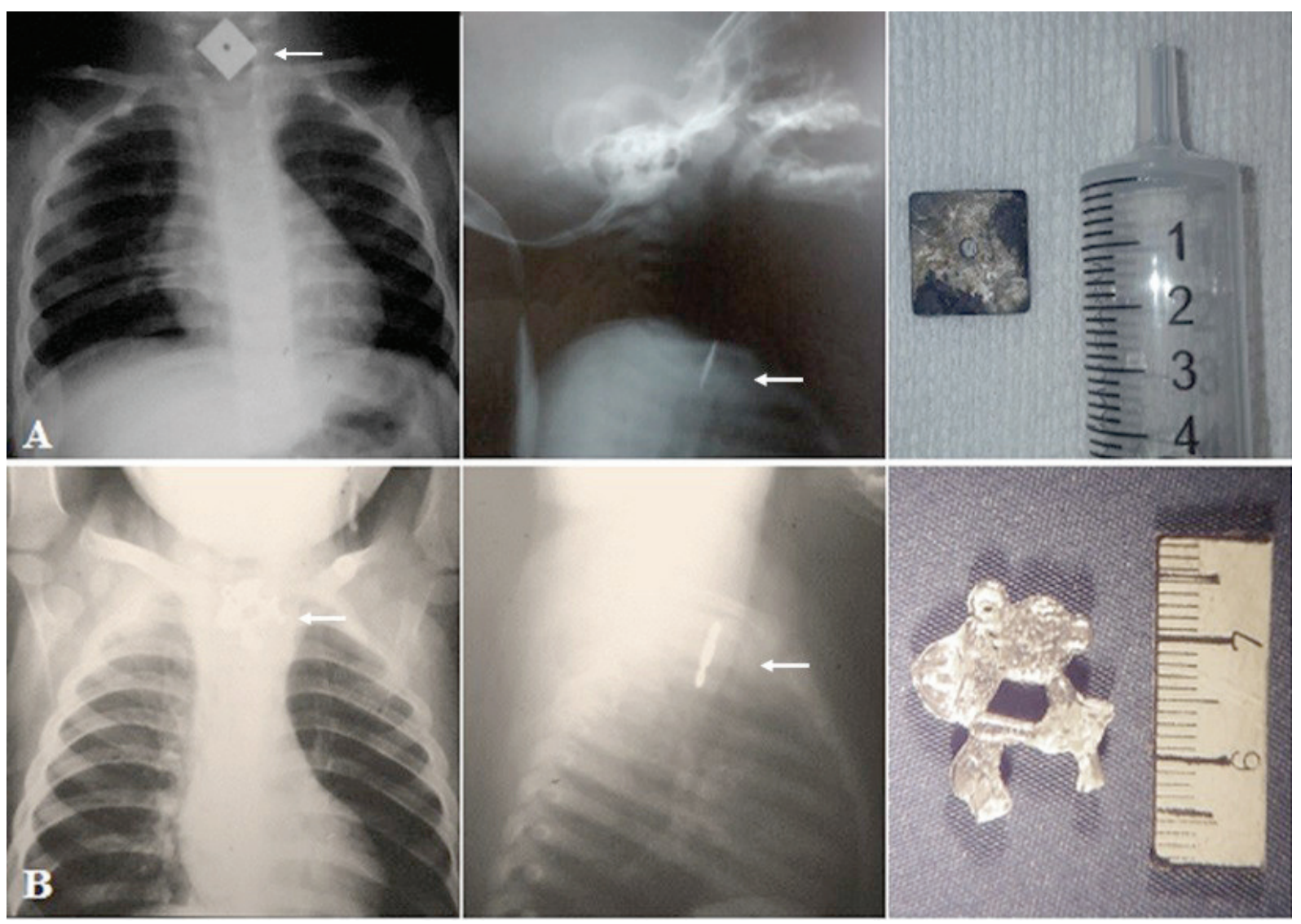


después del procedimiento endoscópico. Fueron alimentados por vía oral, recibieron tratamiento con corticoides orales por 72 horas para disminuir la inflamación periesofágica y fueron dados de alta a las 24 horas.

\section{DISCUSIÓN}

La ingestión de un CE es un problema frecuente y potencialmente grave en pediatría. Los niños de 1 a 3 años de edad son las víctimas prevalentes por diferentes razones: la exploración del ambiente por medio de la boca, la deglución inmadura, la propensión de los niños a la distracción y a jugar mientras comen, y la falta de capacidad cognitiva para distinguir objetos comestibles de los que no lo son. ${ }^{6,7}$

Si bien la ingestión de un CE es común en los niños, los síntomas respiratorios por un CE en el esófago son raros. ${ }^{5,8}$ Cuanto mayor es el tiempo de permanencia de un CE en el esófago, mayor es la

Figura 2. Paciente $n^{\circ}$ 3. Radiografía simple de tórax. A. Vista anteroposterior. Moneda en el esófago (flecha). B. Vista lateral. Compresión traqueal (flecha)

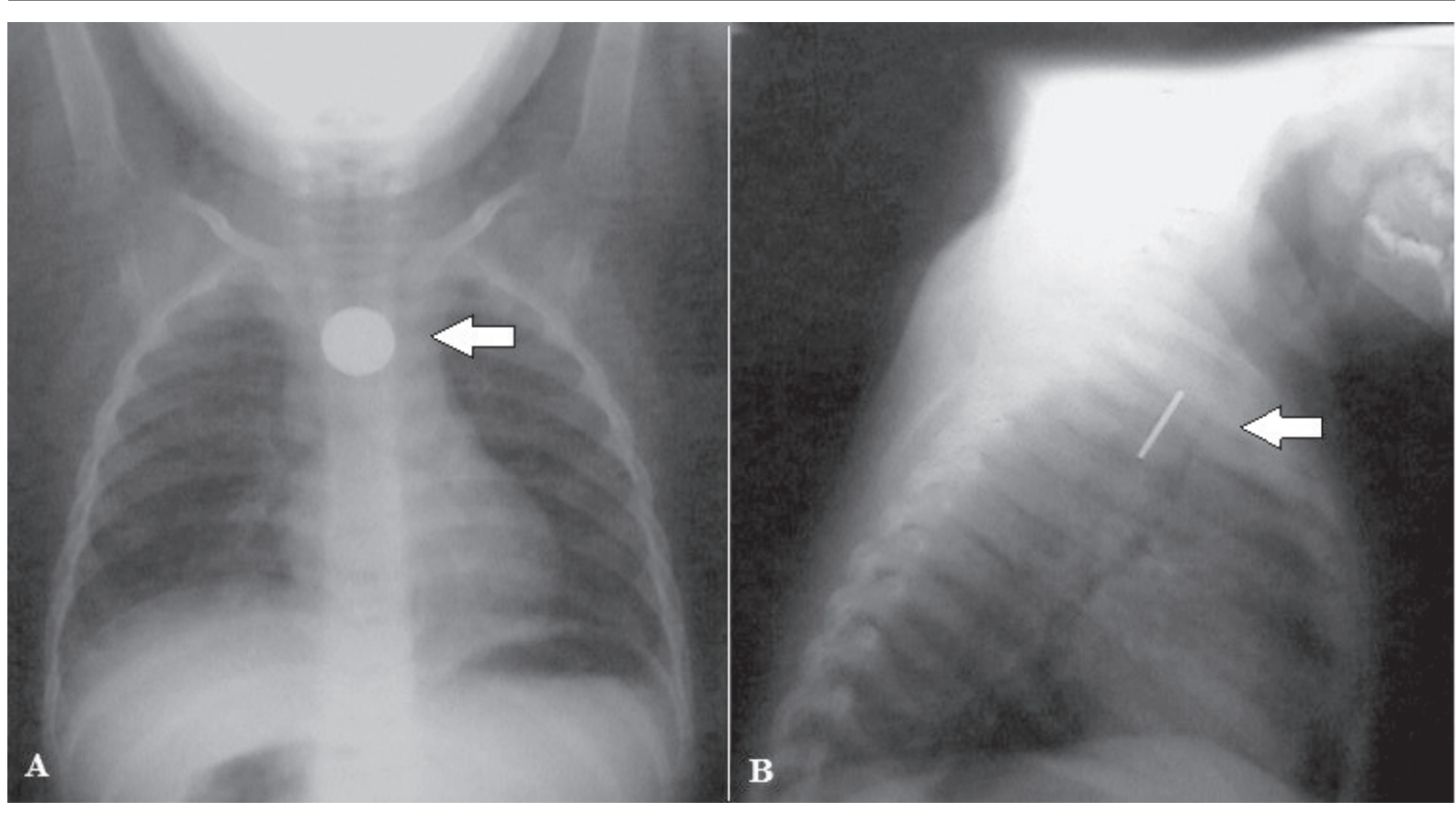

Figura 3. Paciente $n^{\circ}$ 1. A y B. Imágenes endoscópicas. A. Compresión de la pared posterior de la tráquea. B. Esofagoscopía. Cuerpo extraño (arandela) enclavado en la pared anterior del esófago (flecha). El cuerpo extraño no ocluye la luz esofágica (estrella)

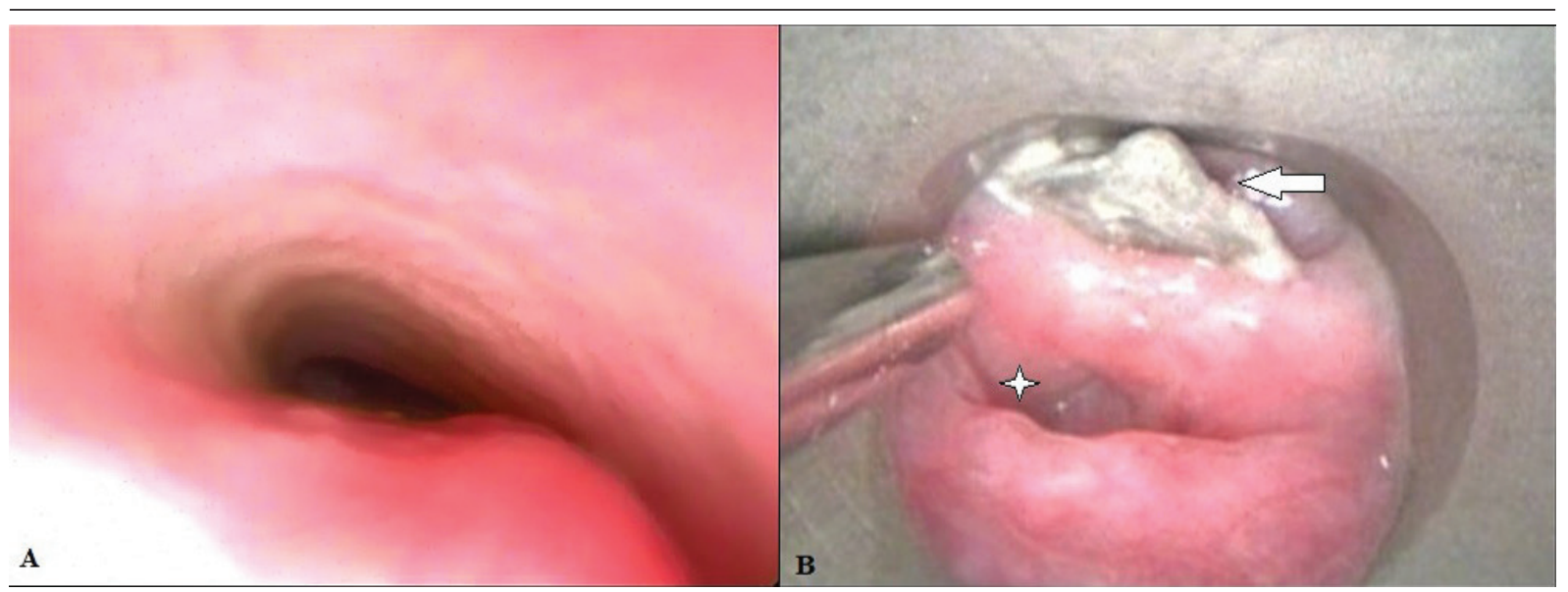


incidencia de los síntomas respiratorios. ${ }^{9}$

La mayoría de los niños con CE esofágicos consultan a un centro asistencial dentro de las 24 horas del impacto del CE por sialorrea y disfagia. ${ }^{6,10}$ En aproximadamente el $80 \%$ de los casos, hay testigos de la ingestión. ${ }^{8,10}$ Los objetos alojados en el esófago dan síntomas que son permanentes desde el momento de la ingesta hasta su extracción y el niño que no come suele ocasionar más preocupación en los padres que el que padece tos. ${ }^{1}$

Un CE crónico en el esófago, es decir, retenido, por lo menos, 1 semana, es poco común. ${ }^{10}$ Se presenta diferente que el agudo, y los síntomas respiratorios son más frecuentes que los gastrointestinales. ${ }^{10,11}$ En un estudio, se observó que el $8 \%$ de los CE esofágicos eran crónicos y, de estos, el $76 \%$ se presentó, principalmente, con síntomas respiratorios, tales como estridor, tos, disfonía y dificultad respiratoria. ${ }^{10}$ Un CE crónico en el esófago puede causar retraso del crecimiento y neumonías recurrentes. ${ }^{12}$

La presentación inusual de un CE esofágico con síntomas respiratorios en lugar de dificultad en la deglución puede explicar el hecho de que permanezca en el esófago durante períodos prolongados. La ingestión de un CE radiolúcido y la ausencia de testigos incrementan, también, el riesgo de CE crónico. ${ }^{8,11,13}$

Los pediatras deben tener en cuenta que un CE esofágico puede dar lugar a síntomas atípicos que simulan asma, crup, bronquitis o bronconeumonía, principalmente, en niños pequeños.

Varios mecanismos han sido propuestos para explicar los síntomas respiratorios debido a un CE esofágico.

La mayoría de los CE esofágicos quedan retenidos a nivel del músculo cricofaríngeo. ${ }^{6}$ La proximidad de la vía aérea al esófago parcialmente obstruido predispone a la aspiración traqueal y a la neumonía. ${ }^{7}$

También los síntomas respiratorios pueden ser secundarios a la compresión traqueal por la presión directa de la pared posterior membranosa de la tráquea (que es particularmente blanda en infantes y niños pequeños) por el propio $\mathrm{CE}$ o por la dilatación esofágica consecuente y a la extensión directa de la reacción inflamatoria a la laringe y la tráquea. ${ }^{7}$ Los $C E$ crónicos pueden producir una inflamación periesofágica o pueden enclavarse en la pared del esófago y producir granulomas que resultan en la compresión traqueal. ${ }^{7}$ Con menor frecuencia, se ha documentado la parálisis cordal secundaria a un CE impactado en el esófago que ocasiona la compresión o el estiramiento de los nervios recurrentes. ${ }^{79}$ Un CE grande, de forma irregular o de bordes afilados puede erosionar la mucosa y crear una fístula traqueoesofágica, incluso atravesarla y ocluir la vía aérea. ${ }^{7}$

Tanto los síntomas respiratorios como los digestivos aumentan durante las comidas. Esto puede deberse al impacto de la comida sobre el $\mathrm{CE}$, al dolor asociado al aumento del peristaltismo esofágico y a la mayor compresión de la vía aérea con el pasaje del bolo alimenticio. ${ }^{10}$

Cuando se sospecha un CE en el esófago, se debe realizar una radiografía simple de tórax y cuello, visión de frente y perfil, ya que, aproximadamente, el 63\%-85\% de los CE son radiopacos. ${ }^{2,10}$ La radiografía puede confirmar la localización, el número de $\mathrm{CE}$ ingeridos, el tamaño y la forma del objeto y la presencia de complicaciones. Si el CE es radiolúcido, el esofagograma puede ponerlo de manifiesto. ${ }^{1,6}$ Otras modalidades de imagen descritas para identificar los CE radiolúcidos o sus secuelas son la tomografía computada y la resonancia magnética. ${ }^{11}$

La mayoría de los CE ingeridos son monedas $(75 \%) .{ }^{1,10}$ Les siguen en frecuencia partes de juguetes, joyas, huesos, pila botón, entre otros. ${ }^{14}$ Es fundamental realizar el diagnóstico diferencial entre una moneda y una pila botón, ya que esta última requiere su extracción urgente, debido a la precocidad de las lesiones que produce. La presencia de doble contorno, en la visión anteroposterior, y el escalón, en el perfil, son indicativos de que se trata de una pila botón. ${ }^{1}$

En caso de localización atípica del objeto impactado, se debe realizar, posteriormente, un esofagograma para descartar una patología subyacente (estenosis esofágica). ${ }^{6}$

La demora diagnóstica mayor de 24 horas aumenta el riesgo de complicaciones, que incluyen erosión o perforación esofágica, mediastinitis, formación de fístulas (traqueoesofágica, aortoesofágica), granulomas y compromiso respiratorio. 8,15

La endoscopía rígida bajo anestesia general es el tratamiento de elección de los CE esofágicos en pediatría. ${ }^{1,6}$ Permite una mejor exposición del CE y emplear pinzas de diferentes tamaños. Se recomienda dentro de las 24 horas del accidente para realizarla en condiciones óptimas, excepto en situaciones de emergencia: dificultad respiratoria de inicio agudo, CE afilado y pila botón.,12

Si bien es cierto que la disfagia es el síntoma de presentación más común de un CE en el esófago, 
los niños con un CE esofágico crónico pueden presentar síntomas respiratorios inespecíficos y no explicables, sin evidencia de disfagia. Los pediatras deben considerar esta posibilidad como parte del diagnóstico diferencial cuando otra etiología no es aparente, principalmente, en niños menores de 3 años. ${ }^{15}$

Es fundamental que los profesionales de la salud alerten a los padres de los riesgos de la ingestión de un CE, los signos y síntomas y la necesidad de atención médica inmediata. ${ }^{14}$

\section{REFERENCIAS}

1. Rodríguez H, Cuestas G, Botto H, Nieto M, et al. Cuerpos extraños en el esófago en los niños. Serie de casos. Arch Argent Pediatr 2013;111(3):e62-5.

2. Sink JR, Kitsko DJ, Mehta DK, Georg MW, et al. Diagnosis of pediatric foreign body ingestion: Clinical presentation, physical examination, and radiologic findings. Ann Otol Rhinol Laryngol 2016;125(4):342-50.

3. Oyewole M, Ajayi O, Hilger A. Oesophageal foreign body: an unusual presentation. J Laryngol Otol 2013;127(12): 1242-3.

4. Liew Z, McKean MC, Townshend J, O'Brien CJ, et al. Oesophageal foreign body presenting with stridor associated with feeding. Arch Dis Child 2013;98(5):384-5.

5. Parray T, Shah S, Apuya JS, Shah S. Atypical presentation of an impacted radiolucent esophageal foreign body. $J$ Anesth 2010;24(5):793-6.

6. Rodríguez H, Passali GC, Gregori D, Chinski A, et al. Management of foreign bodies in the airway and oesophagus. Int J Pediatr Otorhinolaryngol 2012;76(Suppl 1):S84-91.

7. Garg A, Wadhera R, Gulati S, Ghai A, et al. Esophageal foreign body presenting as repiratory distress. Internet $J$ Pediatr Neonatol 2008;11(1):1-3.

8. Hiejima E, Nakase H, Uemoto S, Heike T. Esophageal foreign body causing sustained stridor in an infant. Clin J Gastroenterol 2012;5(2):46-9.

9. Virgilis D, Weinberger J, Fisher D, Goldberg S, et al. Vocal cord paralysis secondary to impacted esophageal foreign bodies in young children. Pediatrics 2001;107(6):e101.

10. Miller RS, Willging JP, Rutter MJ, Rookkapan K. Chronic esophageal foreign bodies in pediatric patients: a retrospective review. Int I Pediatr Otorhinolaryngol 2004;68(3):265-72.

11. Roby BB, Zapapas LM, Fenton LZ, Arganbright JM, et al. Chronic stridor in a nine-month-old. Consider esophageal foreign body. Int J Pediatr Otorhinolaryngol Extra 2016; 13:26-9.

12. Singh A,PandaSS,PandaM,Srinivas M.Upperoesophageal foreign body with acute respiratory distress. BMJ Case Rep 2013;2013:bcr-2013010342.

13. Haegen TW, Wojtczak HA, Tomita SS. Chronic inspiratory stridor secondary to a retained penetrating radiolucent esophageal foreign body. J Pediatr Surg 2003;38(2):e6.

14. Urkin J, Bar-David Y. Respiratory distress secondary to esophageal foreign body: A case report. Scientific World Journal 2006;6:16-9.

15. Liming BJ, Fischer A, Pitcher G. Bronchial compression and tracheosophageal fistula secondary to prolonged esophageal foreign body. Ann Otol Rhinol Laryngol 2016;125(12):1030-3. 